Stellar Seismology 


\title{
White Dwarf and Pre-White Dwarf Oscillations
}

\author{
Arthur N. Cox \\ Los Alamos Astrophysics
}

\begin{abstract}
Compact stars that result from extreme mass loss on the asymptotic giant branch and planetary nebula formation are observed to pulsate in a very large surface effective temperature range as they cool to become the classical white dwarfs. The hottest and most luminous of these display periods in excess of 1000 seconds because they are large, but when the stars arrive on the cooling line on the Hertzsprung-Russell diagram, their periods become generally less than 1000 seconds. Then the stars have masses near $0.6 M_{\odot}$ and radii near $10^{9} \mathrm{~cm}$. Their luminosity depends then almost entirely on the surface effective temperature as the entire star with its legacy of complicated internal luminosity peaks cools to the classical simple electron degenerate structure. Very thin surface layers of hydrogen and helium cover the bulk of the carbon- and oxygen-rich mass that results from hydrogen and helium burning in earlier intermediate mass stellar evolution. The cause of the nonradial pulsations of low angular degree, but rather high radial order, for the most luminous of these stars is the cyclical ionization of carbon and oxygen in layers not too deep that their effectiveness is limited by a long luminosity time scale. Thus the surface hydrogen and helium must be thin, probably thinner than the current period spacings interpretation for PG 1159-035 suggests. For the classical DBV and DAV pulsators, it appears that neither the hydrogen ionization $\kappa$ and $\gamma$ effects or convection blocking at the bottom of a hydrogen convection zone can destabilize the observed pulsations when the overriding short time scale effects of time-dependent convection are included. It appears, however, that a thin CO convection shell can produce pulsations by its time-dependent effects, but again only very thin $\mathrm{H}$ and He surface layers are allowed. This new pulsation mechanism can alleviate the serious problem that DAV variables are observed hotter than the hydrogen $\kappa$ and $\gamma$ effects and convection blocking can predict. The appearance of non-pulsators in the DAV and DBV instability strips can be explained by a too-thick hydrogen and helium surface layer that interferes with (poisons) the $\mathrm{CO}$ ionization convection zone. Finally time-dependent convection predicts that only a few of the many possible modes exist due to their internal amplitude structure that can result in both strong driving and strong damping. Thus actually observed pulsating modes can assist in mapping individual internal white dwarf composition structures, not only by their periods but also the fact that they pulsate.
\end{abstract}




\section{Pre-white dwarf evolution}

After asymptotic giant branch evolution for intermediate mass stars with efficient mass loss, the $\mathrm{CO}$ core resulting from $\mathrm{He}$ burning is almost exposed with only a thin layer of primordial $\mathrm{H}$ and of He produced from earlier stages of shell $\mathrm{H}$ burning. Some of the envelope evaporates to create a very compact pre-white dwarf with a planetary nebula surrounding it. When this nebula blows away, the star is small, with its deep He still creating nuclear energy. Its surface temperature can be over

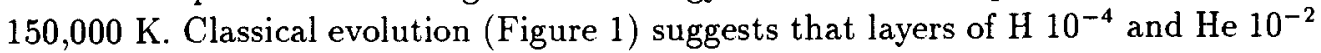
of the mass thick comprise the envelope. More mass loss is being investigated now. The high gravity may not be as inhibiting as earlier thought. Pulsation theory, now including time-dependent convection effects, shows that such thick layers will not give linear theory instability for the observed nonradial modes. For the hottest pre-white dwarfs, this $\mathrm{H}$ and He layer cannot be any thicker than $10^{-9}$ of the stellar mass to pulsate, but the many non-pulsators, with $\mathrm{H}$ and He poisoning in the $\mathrm{CO}$ pulsation driving layers, can have these evolution suggested thick layers.

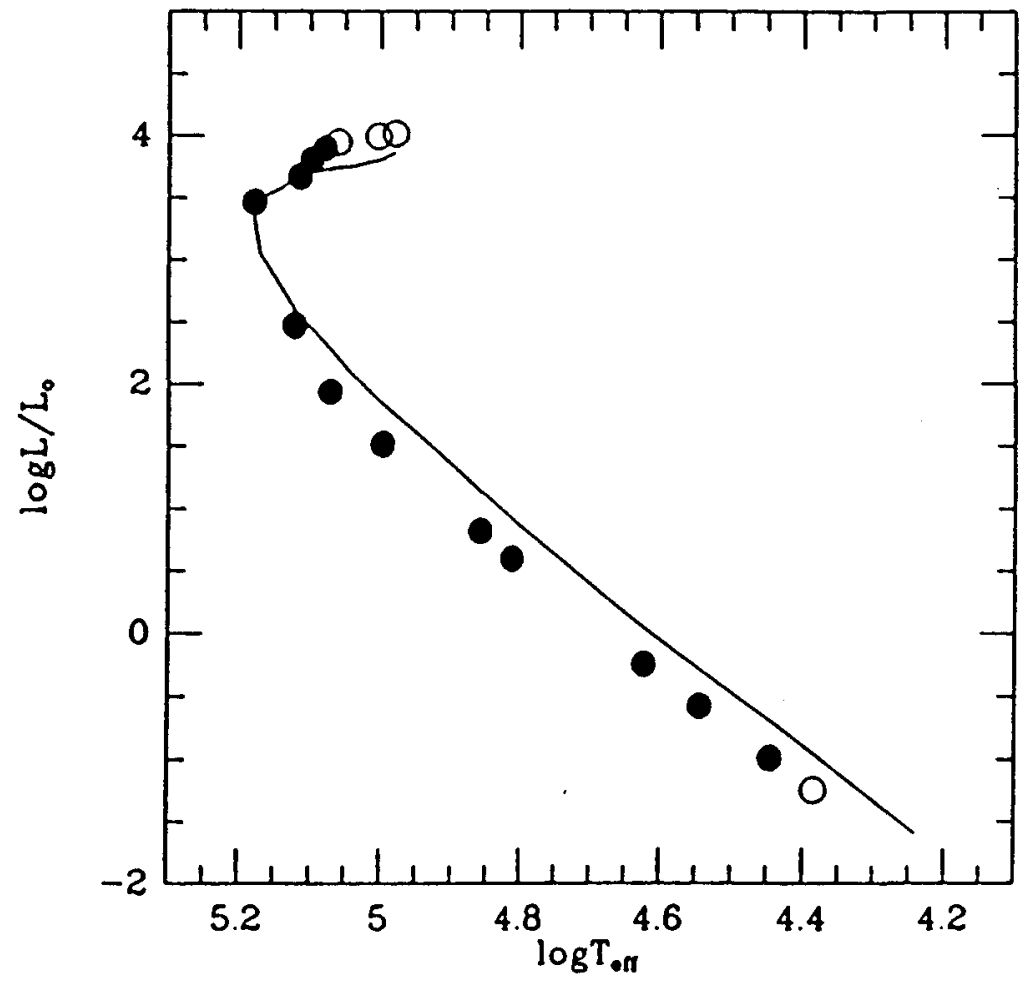

Figure 1 The evolution track for Iben-Hollowell $0.6 M_{\odot}$ models with standard surface $\mathrm{H}$ and $\mathrm{He}$. Solid dots are for pure $\mathrm{CO}$ models that are pulsationally unstable, while the open circles indicate stable models. 


\section{The three classes of white dwarfs}

Various considerations indicate that all these stars have a mass near 0.6 $M_{\odot}$. The most luminous pre-white dwarfs are named the DOV stars. These multiperiodic variable stars are observed with periods of from 500 to over 1500 seconds at surface temperatures over $100,000 \mathrm{~K}$. They have significant surface $\mathrm{CO}$ with rather little $\mathrm{H}$ and $\mathrm{He}$. Due to rapid diffusive settling of $\mathrm{CO}$ and floating of $\mathrm{H}$ and $\mathrm{He}$, the surface compositions probably do not reflect the composition in mass layers $10^{-9}$ deep where the cyclical ionizations of $\mathrm{CO}$ produce pulsational instability by the classical $\kappa$ and $\gamma$ effects. Stars on the evolution track across the top of the Hertzsprung-Russell diagram are larger than those on the cooling track and thus have larger periods for their high radial order nonradial modes. Pure $\mathrm{CO}$ models pulsate over most of this post-planetary nebula state, but the presence of $\mathrm{He}$ in the pulsation driving region dilutes (poisons) the $\kappa$ and $\gamma$ effects driving, and pulsations then occur only in a smaller temperature range. The blue edge for any pulsation is at about $150,000 \mathrm{~K}$. Red edges are determined when the pulsation driving effects layers retreat to deep, adiabatic regions. Recent theoretical work is reported by Stanghellini et al. (1991).

DBV white dwarfs with essentially pure He at their surfaces and in the pulsation driving region pulsate in a narrow temperature range of about $20,000 \mathrm{~K}$ to 25,000 $\mathrm{K}$. Periods are from about 200 to about 1000 seconds. The driving mechanism for these pulsations is related to the deep convection zone in the He. A mechanism called convection blocking periodically valves the luminosity at the bottom of the convection zone at about $10^{-13}$ of the mass deep in a similar way that the classical $\kappa$ and $\gamma$ effects do to convert the emergent luminosity to pulsation motions. Time-dependent convection destroys much of this surface convection driving, but other contributions by the classical $\kappa$ and $\gamma$ effects caused by He ionization $10^{-14}$ of the mass deep and also time-dependent convection in a deep convecting $\mathrm{CO}$ shell about $10^{-6}$ of the mass deep seem to add enough additional driving to produce the observed instability effective temperature band. It has been predicted by two independent groups that a very thin non-convecting $\mathrm{H}$ layer $10^{-15}$ thick can exist on top of these stars to disguise their true DBV character and make an apparent hot DA star pulsate. The instability strip red edge is due to deep convection that mixes $\mathrm{He}$ into the $\mathrm{CO}$, forcing the pure $\mathrm{CO}$ core surface so deep that no convection and its time-dependent effects occur in that material.

The classical DA pulsating white dwarfs have periods between about 200 and 1200 seconds. They have surface effective temperatures between about $11,500 \mathrm{~K}$ and $13,500 \mathrm{~K}$. Recent stellar atmosphere studies including convection in the atmosphere may reduce these temperatures somewhat. Just as for the DBV variables, the DAV pulsators are driven at the bottom of a convection zone about $10^{-13}$ of the mass deep. Very efficient convection is needed to get the convection zone deep enough, and no nonadiabatic calculation has yet predicted pulsations at the observed blue edge of the instability strip. Time-dependent convection destroys much of the convection 
blocking driving, but small amounts of classical $H$ ionization $\kappa$ and $\gamma$ effects $10^{-14}$ of the mass deep and time-dependent convection driving in $\mathrm{CO}_{10}^{-6}$ deep can possibly make the blue edge DA stars pulsate. The instability strip red edge is caused by deep convection that mixes just a little $\mathrm{He}$ into the $\mathrm{CO}$, but an insignificant amount of $\mathrm{CO}$ to the surface, forcing the pure $\mathrm{CO}$ core surface so deep and hot that no convection and its pulsation driving effects occur in that material. Some recent nonadiabatic stability calculations are given by Cox et al. (1987).

\section{Pulsation mechanisms for pre-white dwarfs}

The $\kappa$ effect depends on a large positive logarithmic derivative of the opacity with respect to temperature as shown in Figure 2 for a DOV model.

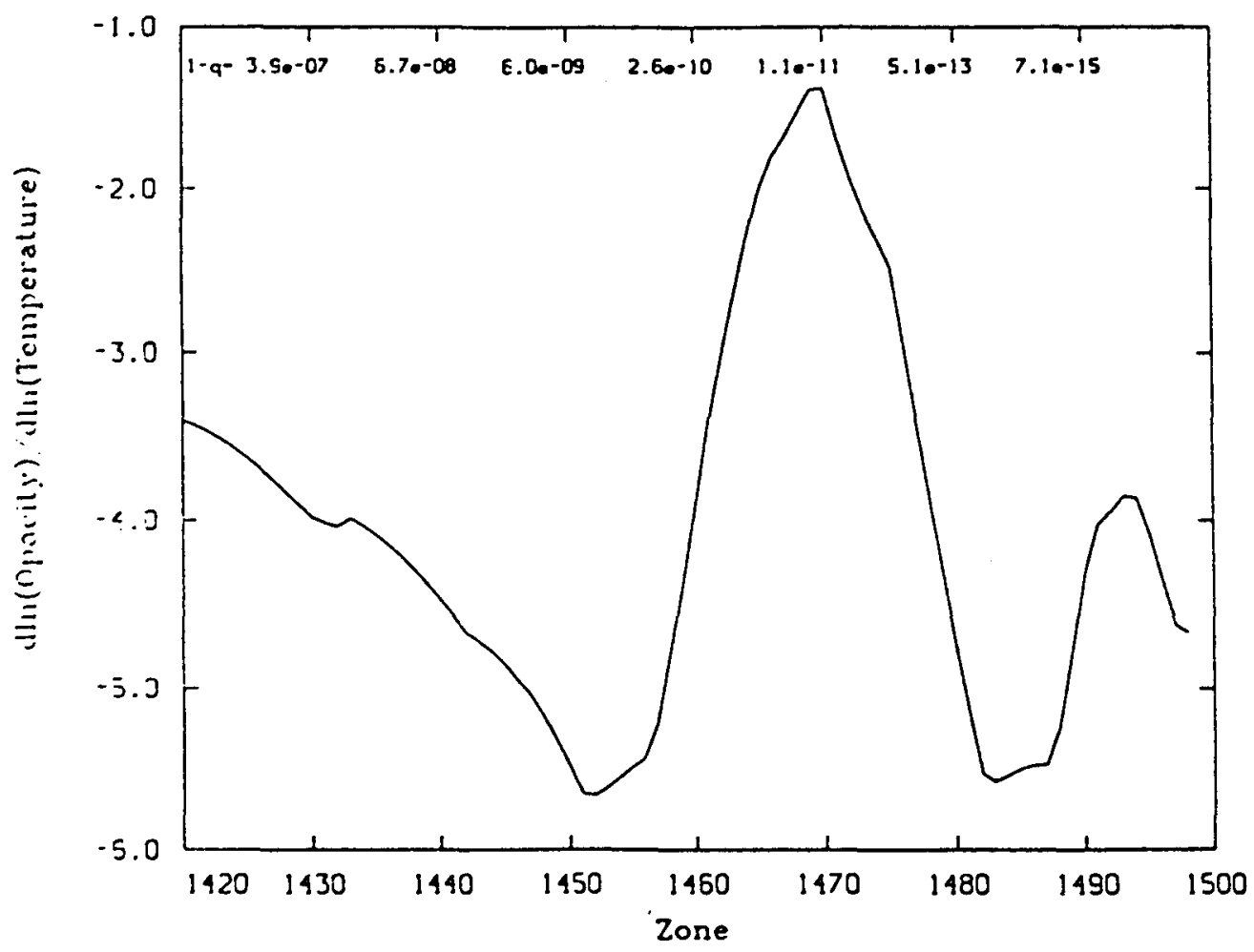

Figure 2 The logarithmic derivative of the opacity with respect to the temperature versus zone number for a pre-white dwarf model at $64,780 \mathrm{~K}$ with a surface and pulsation region composition of half $\mathrm{He}$ and half $\mathrm{C}$.

The $\gamma$ effect can hide luminosity during compression stages when it is small as shown in Figure 3. Figure 4 then shows the work to drive or damp pulsations, and the important region is $10^{-9}$ of the mass deep.

There are two problems with such a thin $\mathrm{H}$ and He layer on top of the pulsation driving CO core. PG 1159-035 is observed to have a period decrease for its strongest 
mode of about $2.5 \times 10^{-11}$ seconds per second, whereas existing evolution calculations (with considerable surface $\mathrm{H}$ and $\mathrm{He}$ ) predict a density deconcentration during this early evolution, and that gives a period increase. Current efforts try to trap modes in rather thick layers $\left(10^{-6}\right.$ of the mass) to predict this observation. Possibly just shrinking of this very hot star now with very little surface $\mathrm{H}$ and $\mathrm{He}$ can more easily allow a correct period decrease prediction.

With only $10^{-9}$ of the mass in the surface layer of $\mathrm{H}$ and He to prevent pulsation poisoning, mode trapping in these layers that can give unequal period spacings may not occur at all. Yet recent observations (Winget et al., 1991) suggest strong mode trapping. I have reviewed the Whole Earth Telescope fourier intensity spectrum kindly supplied by Paul Bradley, and I propose that the largest period spacing fluctuations are incorrect due to wrong mode identifications.

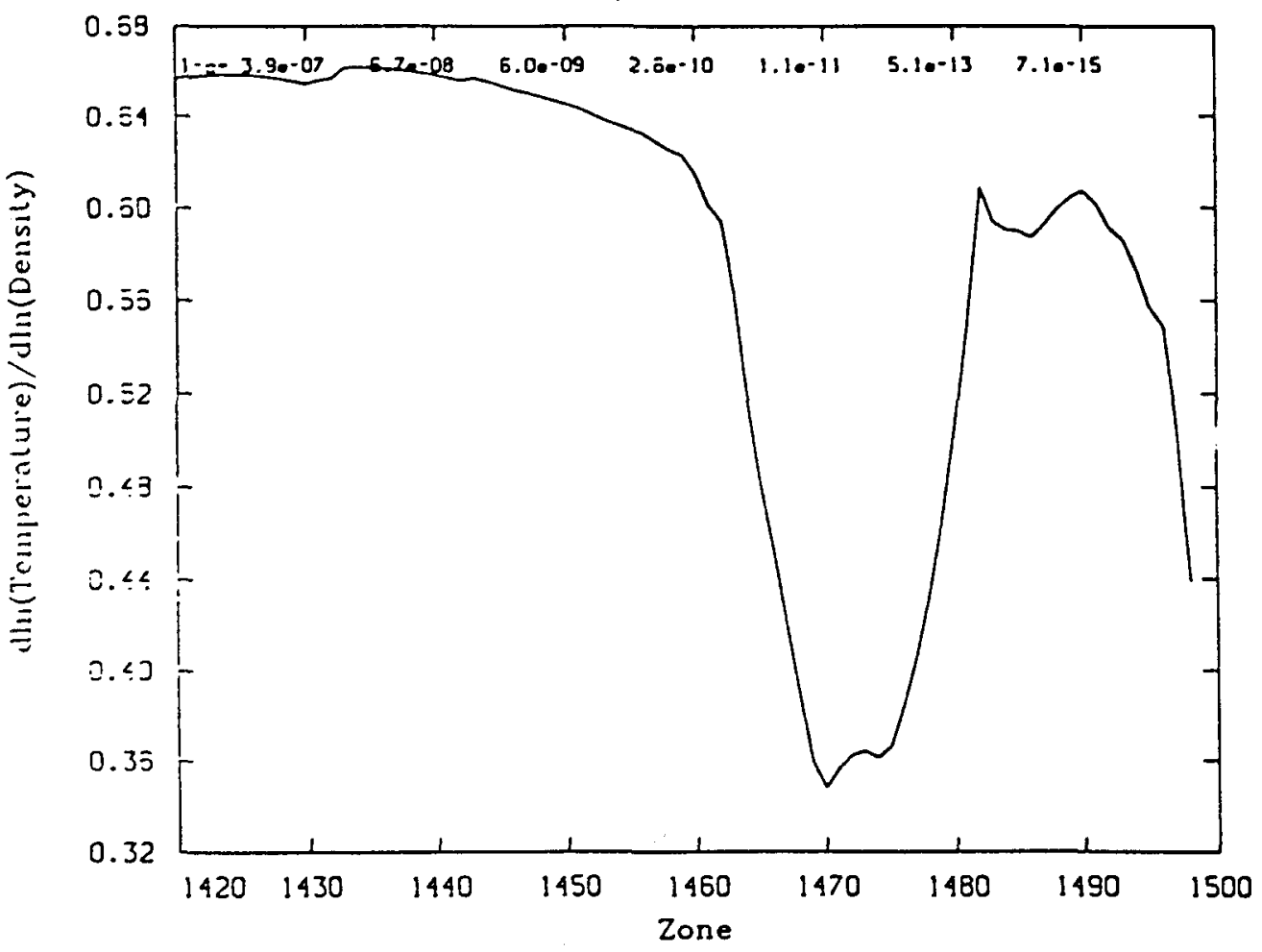

Figure $3 \Gamma_{3}-1$ versus zone number for a pre-white dwarf model at $64,780 \mathrm{~K}$ with a surface and pulsation region composition of half He and half $\mathrm{C}$.

\section{Pulsation mechanisms for DBV white dwarfs}

The pulsation driving region for a $25,000 \mathrm{~K} \mathrm{DBV}$ model is shown in Figure 5 . for the conventional frozen-in convection case. For this model near the DBV instability strip blue edge, driving is mostly the $\kappa$ and $\gamma$ effects in helium, because the surface 
convection is weak with my ratio of mixing length to pressure scale height of 2.5 . There is a slight convection blocking contribution at zone 500 just barely visible. The radiation damping noise between zones 300 and 400 is caused by my three point Lagrangian interpolation, which assures that opacities are accurately interpolated, but there are frequent density and temperature derivative discontinuities.

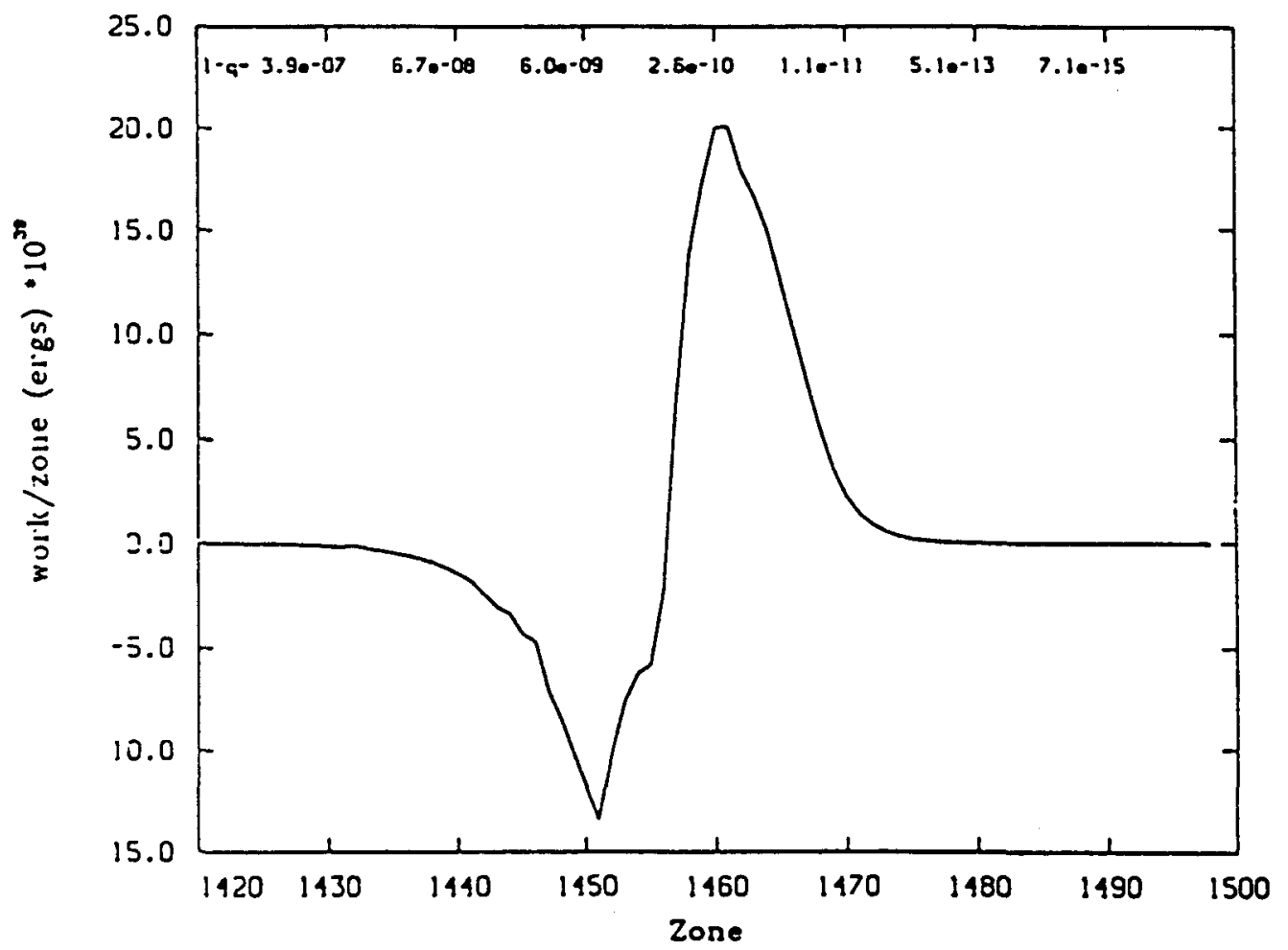

Figure 4 Work versus zone number for a pre-white dwarf model at $64,780 \mathrm{~K}$ with a surface and pulsation region composition of half He and half $\mathrm{C}$. This is for the $g_{14}, \ell=1$ mode at 461 seconds period.

Time-dependent convection using the philosophy of Cox et al. (1966) has been developed for my linear nonadiabatic code. It uses numerical derivatives of the local convection luminosity with respect to the density and temperature on both sides of Lagrangian interfaces and the lagging formulas discussed by Cox and Giuli (1968). When the quickly adapting convection in the surface layers only is considered, most of the radiation blocking is destroyed by convection luminosity, and driving no longer is larger than the deeper damping. This is shown in Figure 6. for the same 25,000 $\mathrm{K}$ model. Figure 7 shows the case when the lagging convection in the deep $\mathrm{CO}$ convecting layer, carrying up to $75 \%$ of the luminosity is allowed. Now net pulsation driving obtains. This lagging results in an amplitude for the convection luminosity variations of only about 0.4 of the instantaneous luminosity, calculated using no 
lagging and the instantaneous configuration. The strong nonadiabatic effects greatly influence the eigensolution.

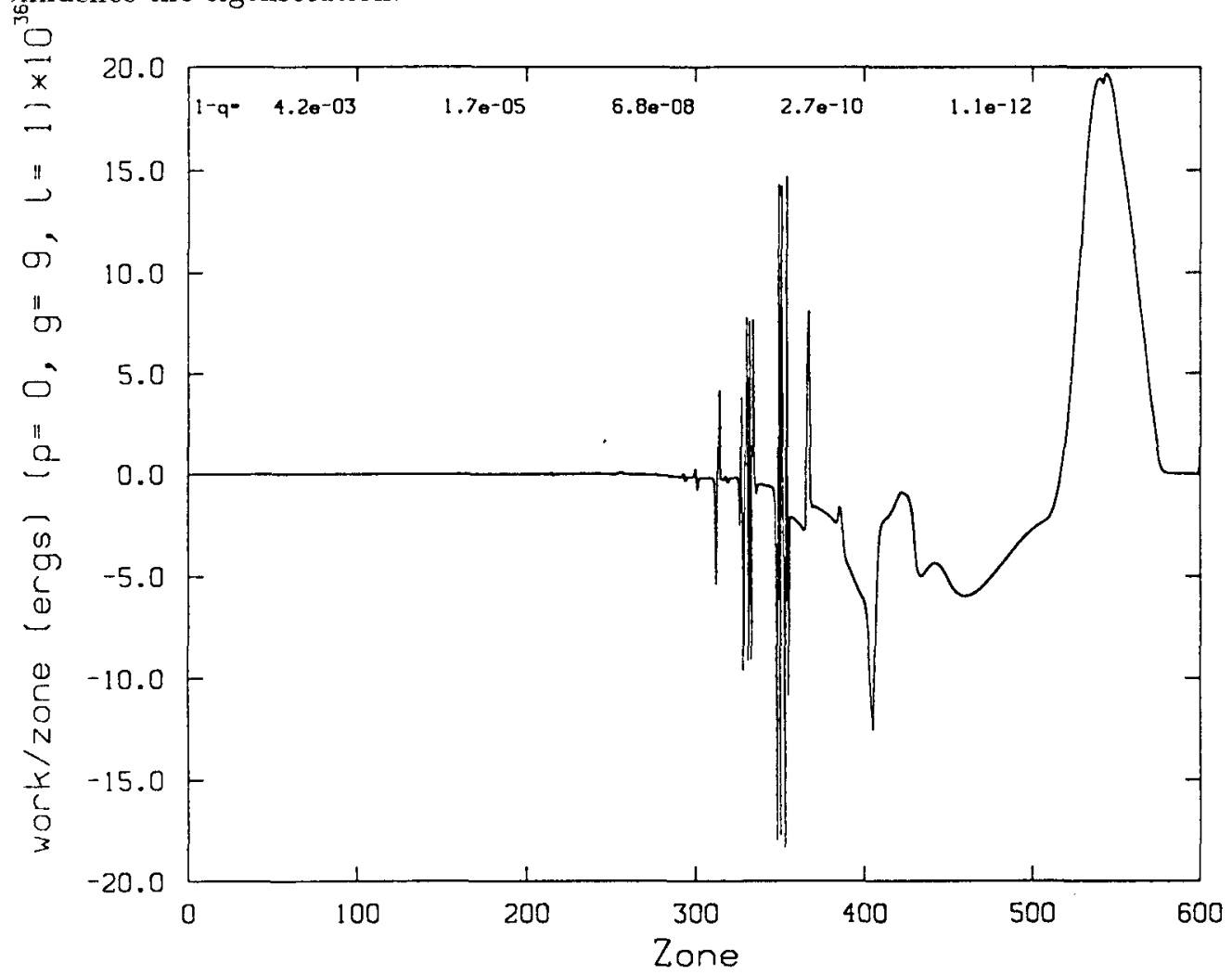

Figure 5 Work versus zone number for a pure He surface and pulsation driving region composition DBV model at $25,000 \mathrm{~K}$. The noise between zones 300 and 400 comes from my three point Lagrangian interpolation in the He opacity table. Zone numbers are used so that eye integration can be made to see if the driving is larger than the damping. This is for the $g_{9}, \ell=1$ mode at 523 seconds period.

Note that it is necessary that there be deep convection to give this time-dependent convection driving and pulsational instability. If the surface He layer is too thick, the transition from surface helium to the $\mathrm{CO}$ core will have a diffusion composition shape instead of a steeper convection overshooting $\mu$ gradient shape. The fact that this DB model is unstable requires that the surface He have a thickness of less than about $10^{-6}$ of the mass. Fortunately, period spacings for $\ell=1$ modes derived by Bradley (private communication) for the DBV variable GD 358 confirm that this He layer thickness is reasonable. When the He thickness gets over $3 \times 10^{-6}$ of the mass, this lower opacity material occupies the region where $\mathrm{CO}$ material would be convecting. 
Then there is no deep convection and probably no pulsations.

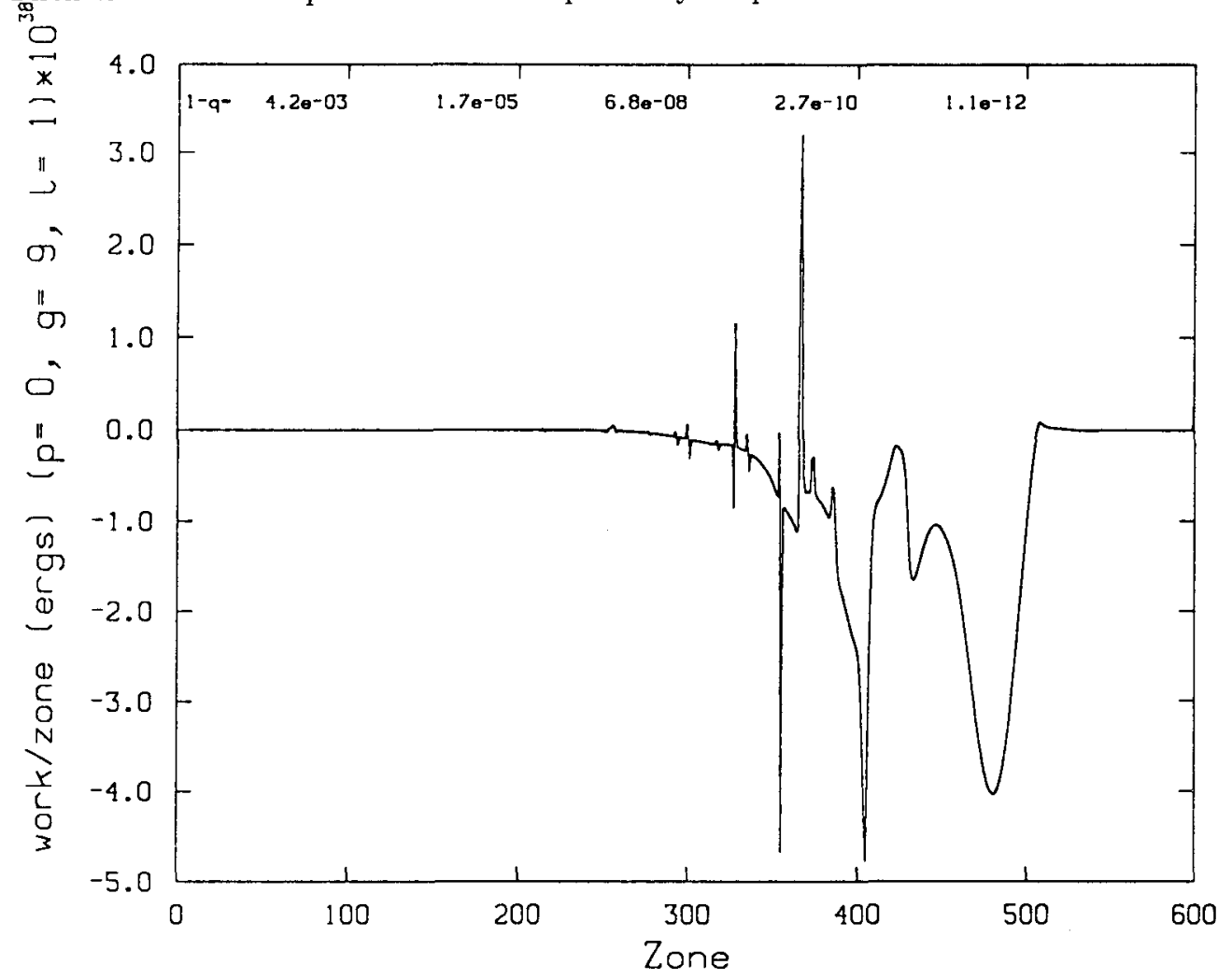

F'igure 6 Work versus zone number for a pure He surface and pulsation driving region composition DBV model at $25,000 \mathrm{~K}$ considering time-dependent convection only in the surface convection zone. This is for the $g_{9}, \ell=1$ mode at 520 seconds period.

\section{DAV white dwarfs and discussion}

The situation is similar for the DAV variables, where frozen-in convection, nonadiabatic calculations do not give instability as hot as $13,500 \mathrm{~K}$. This is true even though Fontaine et al. (1984) inferred instability by only looking at the depth of the convection rather than its strength and ability to block luminosity during compression stages. Agair time-dependent convection destroys almost all surface driving (unless inhibited by strong magnetic fields), but driving in a CO convecting shell only $3 \times 10^{-6}$ deep on top of the CO core gives unstable modes for models as hot as $13,500 \mathrm{~K}$.

Time-dependent convection damps pulsations when the density and temperature variations increase toward the surface, as in the $\mathrm{H}$ and He ionization layers. However, deeper, below the second node, these variations decrease toward the surface, and they together with the natural convection lagging driving and residual surface driving give adequate pulsation driving for DBV and DAV stars. There could be driving also just below the first node and exterior to the density and temperature variations maximum, 
but are no luminosity blocking mechanisms in this few hundreds of thousand kelvin region.

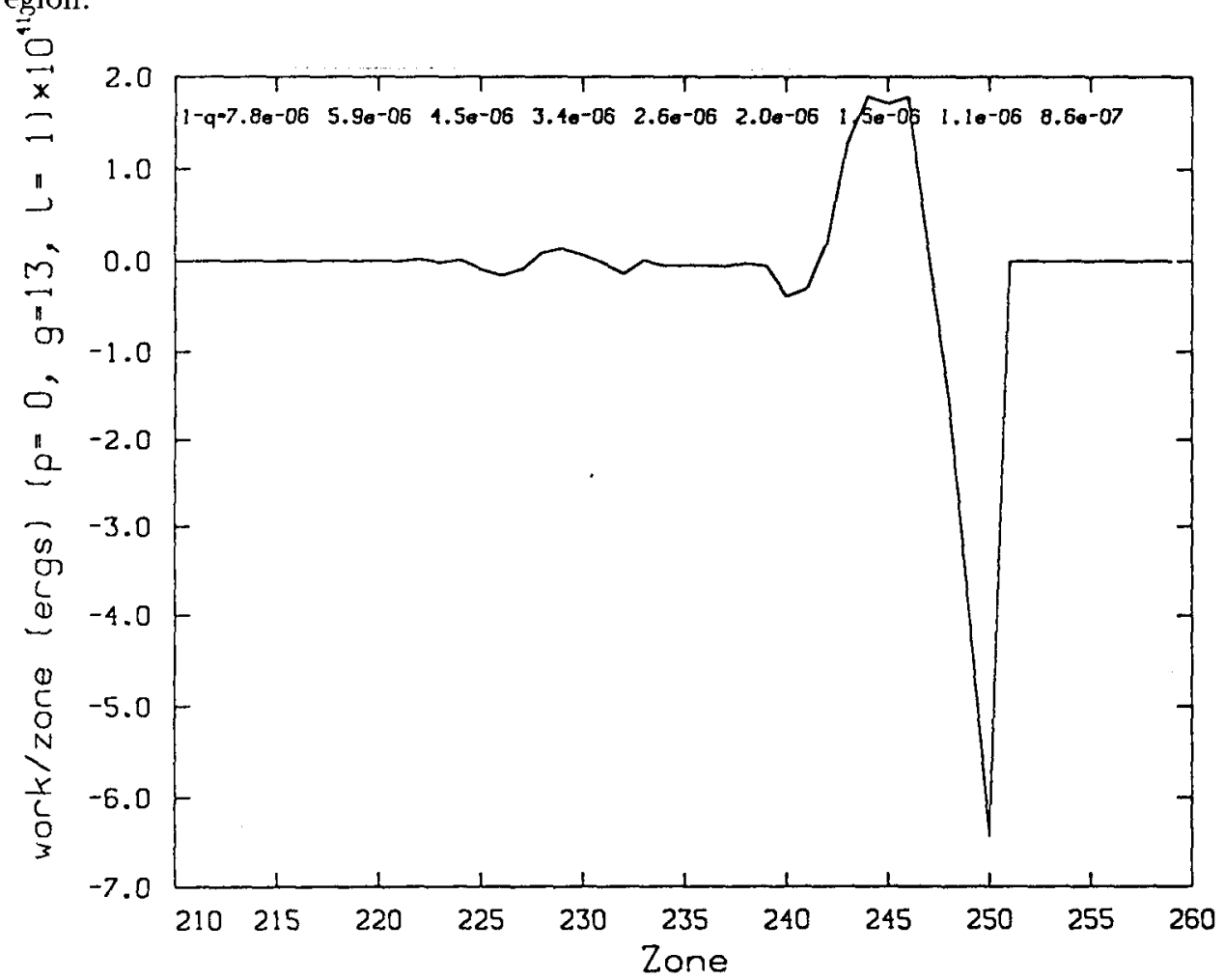

Figure 7 Work versus zone number for a pure He surface composition DBV model at $25,000 \mathrm{~K}$ considering time-dependent convection in the $\mathrm{CO}$ core convection shell that lies between zones 234 and 250 and between 5 and $7 \times 10^{6} K$. This is for the $g_{13}, \ell=1$ mode at 758 seconds period. Not all modes are unstable. Stability depends on the convection derivatives, the convection lagging, and how these influence the eigensolution.

Time-dependent convection gives different drivings from mode to mode. Thus matching observed periods to theoretical ones for various He-CO interface structures allows detailed probing of white dwarfs.

\section{References:}

Cox, A. N., Brownlee, R. R. and Eilers, D. D., 1966, Astrophys. J., 144, 1024.

Cox, A. N., Starrfield, S. G., Kidman, R. B., and Pesnell, W. D., 1987, Astrophys. J., $317,303$.

Cox, J. P. and Giuli, R. T., 1968, "Principles of Stellar Structure" (New York: Gordon and Breach), p. 1045.

Fontaine, G., Tassoul, M., and Wesemael, F., 1984 in, "Theoretical Problems in Stellar Stability and Oscillations," Universite de Liege. p. 328.

Stanghellini, L., Cox, A. N., and Starrfield, S. G., 1991, Astrophys. J., 383, 766.

Winget, D. E., et al., 1991, Astrophys. J., 378, 326. 